\title{
Enzymatic Depletion of the Polysialic Acid Moiety Associated with the Neural Cell Adhesion Molecule Inhibits Antidepressant Efficacy
}

\author{
Steven R Wainwright', Cindy K Barha, ${ }^{2,6}$, Dwayne K Hamson², Jonathan R Epp ${ }^{3}$, Carmen Chow ${ }^{2}$, \\ Stephanie E Lieblich ${ }^{2}$, Urs Rutishauser ${ }^{4}$ and Liisa AM Galea*, I,2,5 \\ 'Graduate Program in Neuroscience, University of British Columbia, Vancouver, British Columbia, Canada; ${ }^{2}$ Department of Psychology, University of \\ British Columbia, Vancouver, British Columbia, Canada; ${ }^{3}$ Program in Neurosciences and Mental Health, Hospital for Sick Children, Toronto, Ontario, \\ Canada; ${ }^{4}$ Department of Cell Biology, Memorial Sloan-Kettering Cancer Center, New York, NY, USA; ${ }^{5}$ Brain Research Centre, University of British \\ Columbia, Vancouver, British Columbia, Canada
}

\begin{abstract}
Antidepressant drugs are too often ineffective, the exact mechanism of efficacy is still ambiguous, and there has been a paucity of novel targets for pharmacotherapy. In an attempt to understand the pathogenesis of depression and subsequently develop more efficacious antidepressant drugs, multiple theories have been proposed, including the modulation of neurotransmission, the upregulation of neurogenesis and neurotrophic factors, normalizing hypothalamic-pituitary-adrenal reactivity, and the reduction of neuroinflammation; all of which have supporting lines of evidence. Therefore, an ideal molecular target for novel pharmaceutical intervention would function at the confluence of these theories. The polysialylated form of the neural cell adhesion molecule (PSA-NCAM) functions broadly, serving to mediate synaptic plasticity, neurogenesis, neurotrophic factor signaling, and inflammatory signaling throughout the brain; all of which are associated with the pathophysiology and treatment of depression. Moreover, the expression of PSA-NCAM is reduced by depression, and conversely enhanced by antidepressant treatment, particularly within the hippocampus. Here we demonstrate that selectively cleaving the polysialic acid moiety, using the bacteriophage-derived enzyme endoneuraminidase $\mathrm{N}$, completely inhibits the antidepressant efficacy of the selective-serotonin reuptake inhibitor fluoxetine ( $F L X)$ in a chronic unpredictable stress model of depression. We also observe a corresponding attenuation of FLX-induced hippocampal neuroplasticity, including decreased hippocampal neurogenesis, synaptic density, and neural activation. These data indicate that PSA-NCAM-mediated neuroplasticity is necessary for antidepressant action; therefore PSA-NCAM represents an interesting, and novel, target for pharmacotherapy.

Neuropsychopharmacology (2016) 4I, I670-1680; doi:I0.I038/npp.20 I5.337; published online I6 December 20 I 5
\end{abstract}

\section{INTRODUCTION}

Multiple classes of antidepressants exist, however, no pharmacological antidepressant has remission rates above $50 \%$, and many patients relapse owing to loss of efficacy (Byrne and Rothschild, 1998). The increasing global burden of depression places a priority on further delineating the mechanisms underlying antidepressant action, and subsequently the exploration of new targets for intervention (Ferrari et al, 2013). Several theories, and lines of evidence, exist regarding the pathogenesis of depression

*Correspondence: Dr LAM Galea, Department of Psychology, The University of British Columbia, 2136 West Mall, Vancouver, British Columbia V6T IZ4, Canada, Tel: + I 250604822 6536, Fax: + I 250 604822 6923, E-mail: Igalea@psych.ubc.ca

${ }^{6}$ Current address: Department of Physical Therapy, Faculty of Medicine, University of British Columbia, Vancouver, BC, Canada; Djavad Mowafaghian Centre for Brain Health, University of British Columbia, Vancouver, BC, Canada.

Received 8 July 2015; revised 4 October 2015; accepted 27 October 2015; accepted article preview online 4 November 2015 and the modes of antidepressant efficacy, including the modulation of neurotransmission, the upregulation of neurogenesis and neurotrophic factors, normalizing hypothalamic-pituitary-adrenal (HPA) reactivity, and the reduction of neuroinflammation (Dantzer et al, 2008; Holsboer, 2000; Wainwright and Galea, 2013). Therefore, an ideal molecular target for pharmaceutical intervention would function at the confluence of these theories.

Chronic stress and the dysregulation of the HPA axis are linked to depression (Anisman and Zacharko, 1982). The hippocampus contains the greatest concentration of glucocorticoid receptors, regulates the HPA axis, and is vulnerable to chronic stress and depression (Sapolsky, 1986). Depressed patients show decreases in hippocampal neurogenesis, neuropil density, and total volume (Boldrini et al, 2009; Stockmeier et al, 2004); conversely, chronic antidepressant treatment is associated with the stabilization of the HPA axis (Ising et al, 2007), increased hippocampal neurogenesis, and greater hippocampal volume (Boldrini et al, 2009; Epp et al, 2013). Capitalizing on this association between stress and 
depression, we used a chronic unpredictable stress (CUS) protocol to induce a depressive-like phenotype in rats, which has good face, construct, and predictive validity (Vollmayr et al, 2007; Wainwright and Galea, 2013; Willner, 2005).

The neural cell adhesion molecule (NCAM) is a protein expressed on the surface of most neural cells and functions to mediate cell-cell interactions (Rutishauser, 2008). NCAM is subject to post-translational modifications, the most prevalent of which is glycosylation by polysialic acid (PSA), forming polysialylated neural cell adhesion molecule (PSA-NCAM). PSA attenuates NCAM-NCAM interactions and interferes with cell adhesion (Rutishauser, 2008). PSA-NCAM is required for proper cell migration, neurite outgrowth, and for activity-induced synaptic plasticity, including LTP; although the ablation of PSA-NCAM does not affect basal synaptic transmission (Burgess et al, 2008; McCall et al, 2013; Muller et al, 1996; Rutishauser, 2008). PSA-NCAM interacts with neuroreceptors (Black et al, 2009; Brezun and Daszuta, 2000; Grzegorzewska et al, 2010), the glucocorticoid receptor (Rodriguez et al, 1998), and with neurotrophic factors directly associated with antidepressant effects such as BDNF via the TrkB and p75 receptors, and FGF receptors 1 and 2 (Aonurm-Helm et al, 2008; Elsayed et al, 2012; Vutskits et al, 2001). PSA-NCAM is a member of the immunoglobulin superfamily and functions in the immune response, directly interacting with protein complexes associated with inflammation and linked to the pathogenesis of depression (Koo et al, 2010; Krushel et al, 1999). It is important to note that the ablation of neurogenesis (Bessa et al, 2009; David et al, 2009; Santarelli et al, 2003), serotonergic neurotransmission (Lieben et al, 2006), or other proteins associated with antidepressant efficacy such as p11 (Egeland et al, 2010) do not induce a depressive-like behavioral phenotype; however the $\mathrm{NCAM}^{-/-}$knockout does indeed produce a depressive-like phenotype (Aonurm-Helm et al, 2008) in an animal model, suggesting a potentially unique contribution to the pathophysiology of depression, and to its treatment. PSA-NCAM is highly expressed by newly generated neurons in the adult hippocampus, as well as at the synapses of mature neurons during times of synaptic plasticity in the hippocampus as well as other brain regions (Rutishauser, 2008). PSA-NCAM is also expressed in multiple regions associated with the regulation of affect, including the hippocampus, prefrontal cortex, amygdala, raphe nucleus, and nucleus accumbens (Sairanen et al, 2007; Wainwright and Galea, 2013). Indeed, the expression of PSA-NCAM is reduced in depressed patients, and in models of depression, and conversely enhanced by antidepressant treatment, in many regions (Sairanen et al, 2007; Varea et al, 2007; Wainwright et al, 2011). The expression of PSA-NCAM is also associated with serotonergic and dopaminergic neurotransmission within these regions (Brezun and Daszuta, 2000; Castillo-Gomez et al, 2008).

In this study, we examined whether PSA-NCAM is required for the antidepressant efficacy of the selectiveserotonin reuptake inhibitor fluoxetine (FLX). PSA-NCAM was selectively depleted using an intracerebroventricular (ICV) infusion of the bacteriophage-derived enzyme endoneuraminidase $\mathrm{N}$ (EndoN) (Rutishauser et al, 1985) prior to the administration of FLX within our CUS model of depression. We then evaluated the requirement of
PSA-NCAM for antidepressant efficacy using standardized neurogenesis-dependent and -independent behavioral measures (David et al, 2009) in addition to measures of neuroplasticity within the hippocampus, including synaptic density, neurogenesis (proliferation, survival, differentiation), and neural activation (using the immediate early gene cFos). Through the direct disruption of the PSA moiety we demonstrated that PSA-NCAM is required for antidepressant efficacy and that in its absence neuroplasticity is attenuated, and the behavioral efficacy of FLX is completely inhibited.

\section{MATERIALS AND METHODS}

\section{Subjects}

Sixty-six male Sprague-Dawley rats (University of British Columbia) weighing $\sim 300 \mathrm{~g}$ upon arrival were used. Rats were pair-housed under standard laboratory conditions $(12: 12 \mathrm{~h}$ light/dark cycle with lights on at $0700 \mathrm{~h}$, at $21 \pm 1^{\circ} \mathrm{C}$, food and water provided ad libitum). Rats were randomly assigned to eight treatment groups according to whether they received ICV infusion of EndoN or saline, and/or received CUS or noCUS, and/or received FLX or vehicle (VEH). The eight groups were: Sal+noCUS+VEH $(n=8)$; Sal+noCUS+FLX $(n=8)$; Sal+CUS+VEH $(n=8) ; \quad$ Sal+CUS+FLX $(n=10) ; \quad$ EndoN+ noCUS+VEH $(n=8)$; EndoN+noCUS+FLX $(n=8)$; EndoN+ CUS+VEH $(n=8)$; EndoN+CUS+FLX $(n=8)$. Sample sizes were chosen from previous studies (David et al, 2009; Santarelli et al, 2003; Wainwright et al, 2011). All procedures were carried out in accordance with the Canadian Council on Animal Care guidelines and were approved by the local Animal Care Committee.

\section{Surgery}

Surgeries were conducted 6-10 days after rats arrived, using aseptic procedures. Under isoflurane anesthesia each rat received either a single, unilateral ICV infusion of EndoN $(1 \mu \mathrm{l} ; 180 \mathrm{U} / \mu \mathrm{l})$ or vehicle $(1 \mu \mathrm{l} ; 0.9 \%$ saline $)$ via a Hamilton syringe (coordinates of AP: $-1, \mathrm{ML}:-1.5, \mathrm{DV}:-3.7$ from bregma). EndoN was prepared and provided by Dr Rutishauer in the method of Hallenbeck et al, (1987). Marcaine $(0.5 \mathrm{mg})$, buprenophrine $(0.02 \mathrm{mg} / \mathrm{kg})$, and ketoprofen $(5 \mathrm{mg} / \mathrm{kg})$ were used as analgesics. Rats were pairhoused and given 6 days for recovery (Figure 1).

\section{BrdU Administration}

One day prior to beginning CUS or noCUS treatment, all rats were given two i.p. injections of 5-Bromo-2-deoxyuridine (BrdU; $200 \mathrm{mg} / \mathrm{kg}$ ) $8 \mathrm{~h}$ apart. The timing was chosen to examine the effects of CUS on neurogenesis independent of the ability of CUS to alter cell proliferation. BrdU (SigmaAldrich, St Louis, MO) was prepared by dissolving $20 \mathrm{mg} / \mathrm{ml}$ in $0.9 \%$ saline buffered with $2 \mathrm{~N} \mathrm{NaOH}$.

\section{CUS Procedure and Antidepressant Treatment}

All rats were subjected to 6 weeks of either CUS or noCUS. The noCUS rats were housed in a separate colony room from rats receiving CUS to eliminate any odor confounds of 


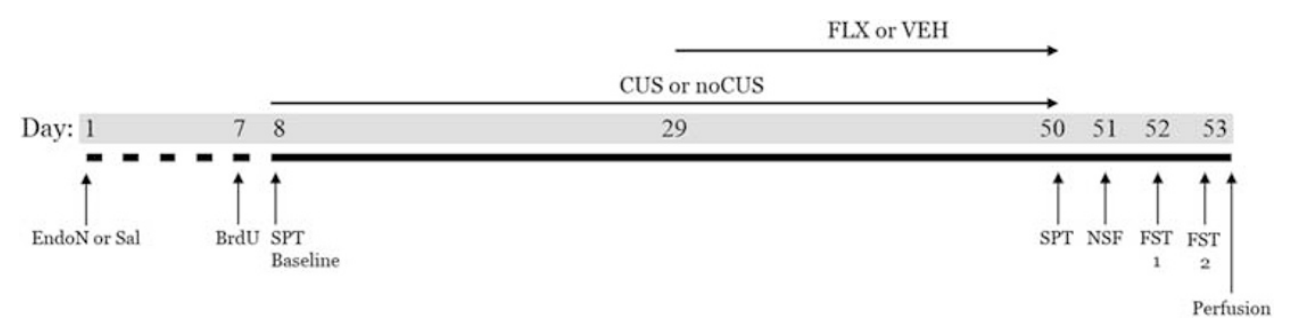

Figure I Experimental timeline.

stressed conspecifics. The CUS protocol consisted of the pseudo-random presentation of two stressors per day (Wainwright et al, 2011). The first 3 weeks of CUS was to develop a depressive-like phenotype, while during the final 3 weeks of CUS (or noCUS) all rats underwent concurrent daily treatment with either FLX $(10 \mathrm{mg} / \mathrm{kg}$; i.p.; in $0.9 \%$ saline and 10\% DMSO) or vehicle and the experimenter was not blind during this process.

\section{Sucrose Preference Test}

For testing each rat was moved to a single cage, food and water deprived for $4 \mathrm{~h}$, then presented with two pre-weighed bottles containing a $1 \%$ sucrose solution or tap water for $1 \mathrm{~h}$ (at $1900 \mathrm{~h}$ ). Sucrose preference was calculated blind as: (sucrose intake/(sucrose intake+water intake) $\times 100$ ). Anhedonia was determined as the proportion of sucrose preference at week 6 against a baseline level taken prior to the commencement of CUS.

\section{Novelty Suppressed Feeding Test}

Rats were food-deprived for $18 \mathrm{~h}$ and then placed in a novel environment with a food pellet in the center of the arena $(50 \times 50 \times 50 \mathrm{~cm})$. The latency to start feeding $(\max 10 \mathrm{~min})$ was measured blind to assess anxiety-like behavior. Following testing, food consumption was measured $(1 \mathrm{~h})$ as a control for appetite differences.

\section{Forced Swim Test}

Stress-coping behavior was assessed using the forced swim test (Wainwright et al, 2011). In brief, rats were placed in a cylindrical glass container filled to a depth of $50 \mathrm{~cm}$ with water at $23 \pm 0.5^{\circ} \mathrm{C}$. The forced swim test was conducted over two sessions $24 \mathrm{~h}$ apart lasting $15 \mathrm{~min}$ (session-1) and 5 min (session-2). Videotaped sessions were scored blind for swimming, climbing, and immobility.

\section{Hormone Assays}

Blood was collected under basal conditions from the tail vein each week from the CUS groups, and only on weeks 1,3 , and 6 from the noCUS groups to avoid additional stress. Perfusion blood was collected for testosterone assay. Samples were stored overnight at $4{ }^{\circ} \mathrm{C}$, centrifuged at $10000 \mathrm{rpm}$ for $15 \mathrm{~min}$, and serum was stored at $-20^{\circ} \mathrm{C}$. Samples were assayed in duplicate using a modified protocol from a commercially available ${ }^{125} \mathrm{I}$ radioimmunoassay kits for testosterone (MP Biomedicals, Costa Mesa, CA) and rat corticosterone (MP Biomedicals, Orangeburg, NY).

\section{Histology}

Rats were anesthetized with a lethal dose of sodium pentobarbital (Euthanyl; Bimeda-MTC, Cambridge, ON) and perfused transcardially $(0.9 \%$ saline followed by $4 \%$ paraformaldehyde) $90 \mathrm{~min}$ after the forced swim test. Brains were extracted and post-fixed with $4 \%$ paraformaldehyde for $24 \mathrm{~h}$, then cryoprotected with $30 \%$ sucrose and stored $\left(4^{\circ} \mathrm{C}\right)$. Brains were sectioned throughout the rostral-caudal extent of the hippocampus into series of $40-\mu \mathrm{m}$ coronal slices. Brain sections were stained for PSA-NCAM (mouse anti-PSANCAM 1:1000; Millipore, Temecula, CA); Ki67, an endogenous marker of cell proliferation (Rabbit anti-Ki67 1:500; Vector Laboratories, Burlingame, CA); cFos, an immediate early gene (rabbit anti-cFos 1:1000; Santa Cruz Biotechnology); PSD-95, an endogenous measure of synaptic density (1:1000; Abcam); BrdU, to assess cell survival (mouse anti-BrdU-1:200; Roche, Indianapolis, IN); or double-labeled BrdU/ NeuN to measure neuron differentiation (mouse anti-NeuN-1:250; Millipore; rat anti-BrdU; AbD Serotech, Oxford, UK). An ABC kit (Vector Laboratories) was used for detection, with diaminobenzadine as the chromogen (Sigma; Oakville, ON, Canada). BrdU/NeuN immunostained sections were incubated with conjugated fluorescent secondary antibodies (BrdU-1:500 donkey antirat Cy3 (Jackson ImmunoResearch, Westgrove, PA); NeuN$-1: 500$ donkey anti-mouse Alexa 488 (Invitrogen, Eugene, OR)).

BrdU, Ki67, cFos and PSA-NCAM immunoreactive (ir) cells were counted blind on an Olympus light microscope at $\times 1000$ magnification in every 10th section throughout the granule cell layer (GCL), including the subgranular zone (SGZ) and hilus. Counts were aggregated and multiplied by 10 to estimate the total number of ir cells, as described (Epp et al, 2010). Counts were separated by the dorsal and ventral hippocampus, as the ventral hippocampus is specifically implicated in depression (Fanselow and Dong, 2010). The percentage of BrdU/NeuN double-labeled cells was obtained by selecting $25 \mathrm{BrdU}$-ir cells per brain arbitrarily from at least five sections per brain. Fluorescent imaging was performed on an Olympus epifluorescent microscope under $\times 400$ magnification, and verified using confocal microscopy. Area measurements of the GCL and hilus were calculated using the program ImageJ (NIH). Volume was estimated using Cavalieri's principle.

The optical density of PSD-95 expression at $\times 40$ magnification was calculated using ImageJ on photomicrographs with fixed light intensity and exposure settings acquired using cellSens software (Olympus Canada, Toronto, ON, Canada). Mean grey levels were measured by pseudorandomly placing open circles along different regions of the 
entire extent of the hippocampus: dentate gyrus (GCL; both supra- and infrapyramidal blades), CA1 (stratum radiatum), and CA3 (stratum lucidum). The background grey level for each section was obtained from the mean of three circles placed within the corpus callosum to control for any potential variations in staining. A threshold was then set at three-times the background mean grey level, and the mean grey level above the threshold for each region was measured, averaging the grey levels of all circles within that region, adapted from (Snyder et al, 2012).

\section{Data Analyses}

Factorial or repeated-measures ANOVA were conducted on each behavioral, neural or endocrine measure with stress (noCUS, CUS), enzyme (Sal, EndoN), and drug (VEH, FLX) as the between-subjects factors. For repeated-measures ANOVA within-subjects factors were: forced swim test behavior (immobility, swimming, climbing), week (baseline, 3 and 6) for hormones, area (GCL+SGZ, hilus) and region (dorsal, ventral) for volume, or region (dorsal, ventral) for density of Ki67-ir, PSA-NCAM-ir, and cFos-ir cells or hippocampal field (DG, CA1, CA3) for PSD-95. Any violations of homogeneity of variance used Hyundt-Feldt corrections (cFos, BrdU) and violations in normality (CORT) were transformed. Subjects were excluded if brain tissue was damaged resulting in missing sections for histology $(n=2-4)$, if performance was either not recorded due to equipment malfunction or deemed an outlier (greater than two SDs $(n=2-4)$, or not enough serum for duplicates/high coefficient of variation $(n=10)$. All post hoc tests utilized Newman-Keuls. We expected EndoN would attenuate antidepressant action and any a priori comparisons were subjected to a Bonferroni correction. Significance level was 0.05 .

\section{RESULTS}

\section{EndoN Treatment Disrupts the Behavioural Efficacy of FLX}

CUS treatment reduced sucrose preference (Figure 2a; main effect of stress $(p<0.005)$ but there were no other significant main or interaction effects (all $p$ 's $>0.11$ ). EndoN treatment showed no independent effect on sucrose preference (main effect of enzyme $p>0.25$ ), suggesting no basal disruption based on enzyme treatment. A priori comparisons were conducted on FLX effects in CUS groups with and without EndoN treatment. FLX increased sucrose preference in CUS rats with no EndoN $(p<0.041)$, whereas EndoN inhibited the effect of FLX treatment on sucrose preference as the EndoN-CUS-FLX group had a lower preference than the Sal-CUS-FLX group $(p<0.04)$.

FLX treatment, regardless of CUS, decreased latency to feed in the novelty-suppressed feeding test but only in the absence of EndoN treatment ( $p$ 's $<0.014$; enzyme by drug interaction $p<0.0005$; Figure 2b). EndoN inhibited the effects of FLX treatment regardless of CUS $(p=0.0002)$, as the EndoN-CUS-FLX and EndoN-noCUS-FLX groups had a significantly greater latency to feed than their controls (Sal-CUS-FLX $(p<0.008)$, and the Sal-noCUS-FLX $(p<0.0005))$. There was also a main effect of enzyme $(p=0.0052)$ but no other significant main or interaction effects ( $p$ 's $>0.14)$. Importantly, there were no significant differences between any of the groups (all main and interaction effects $p$ 's $>0.19$ ) in food consumption following the test indicating no appetite differences between groups.

As expected, CUS increased immobility and decreased swimming behavior, whereas FLX treatment decreased immobility and increased swimming behavior, compared with VEH treatment (Figure $2 c$ and d; all $p$ 's $<0.04$; behavior by stress by drug interaction: $p=0.0018$ ). EndoN functioned to eliminate the effects of FLX treatment on immobility $(p<0.001)$ and swimming $(p<0.0005)$ compared with the saline-infused treatment groups (behavior by enzyme by drug $(p=0.0009)$, behavior by enzyme by stress $(p=0.048))$. There were also significant two-way interactions (behavior by stress: $p<0.0001$; behavior by enzyme: $p=0.006$ ) and a main effect of behavior $(p<0.0001)$ but no other significant main or interaction effects $(p$ 's $>0.16)$.

\section{EndoN Treatment Reduced Neuroplasticity and Attenuated Some FLX-Induced Increases in Neuroplasticity}

As expected, EndoN treatment significantly reduced the density of PSA-NCAM-ir cells across all groups in both the dorsal and ventral dentate gyrus and drug groups, despite the interactions (DG; Figure 3a, e and f; all $p$ 's $<0.0005$; enzyme by region interaction: $p<0.00001$; enzyme by drug interaction: $p=0.021)$. There were also main effects of enzyme and area (both $p$ 's $<0.00001$ ) but no other significant effects ( $p$ 's $>0.16$ ).

Both EndoN and CUS significantly decreased the density of BrdU-ir cells in the GCL+SGZ (Figure 3b; $p<0.0002$ ), but not in the hilus $(p>0.94$; area by enzyme interaction $p<0.00001$; area $\times$ stress interaction $p=0.038)$. There were also significant main effects of stress $(p=0.047)$, enzyme $(p<0.0001)$, region $(p<0.00001$ with greater density of BrdU-ir in the ventral compared with dorsal dentate gyrus) and area $(p<0.0001)$ but no other significant interaction effects (all $p$ 's $>0.09$ ). Furthermore, using a priori comparisons Endo-CUS-FLX had a lower density of BrdU-ir cells than the Sal-CUS-FLX group in both the ventral and dorsal GCL (both $p$ 's $<0.0008$ ). EndoN and CUS both reduced the proportion of BrdU/NeuN co-labeled cells (Figure $3 \mathrm{~g}-\mathrm{k}$ ) in the GCL, with the effect of CUS most pronounced in the dorsal region (region by enzyme by stress interaction $p<0.02)$. FLX treatment increased the density of Ki67-ir cells in the ventral $(p=0.0043)$ but not dorsal $(p<0.89)$ GCL, regardless of CUS or enzyme group (Figure $3 c$; region by drug interaction: $p=0.042$ ). There were no other significant main or interaction effects (all $p$ 's $>0.14$ ).

In response to acute swim stress, CUS groups showed reduced cFos-ir expression (main effect of stress: $p=0.044$ ). FLX reduced the density of cFos-ir cells in the ventral DG $(p=0.002)$, whereas EndoN treatment inhibited the effect of FLX on the density of cFos-ir cells in the ventral DG $(p=0.79$ compared with Veh-Sal, or $p=0.18$ compared with EndoNSal, irrespective of stress conditions; Figure $3 \mathrm{~d}$; region by enzyme by drug interaction: $p=0.022$ ). However, a priori comparisons indicated that these differences were largely driven by effects under CUS conditions with Endo-CUS-FLX significantly greater than Sal-CUS-FLX $(p=0.0002)$, but not Sal-CUS-Veh $(p=0.15)$. There were no other significant effects ( $p$ 's $>0.09)$ except a main effect of region $(p<0.0001)$. 
a

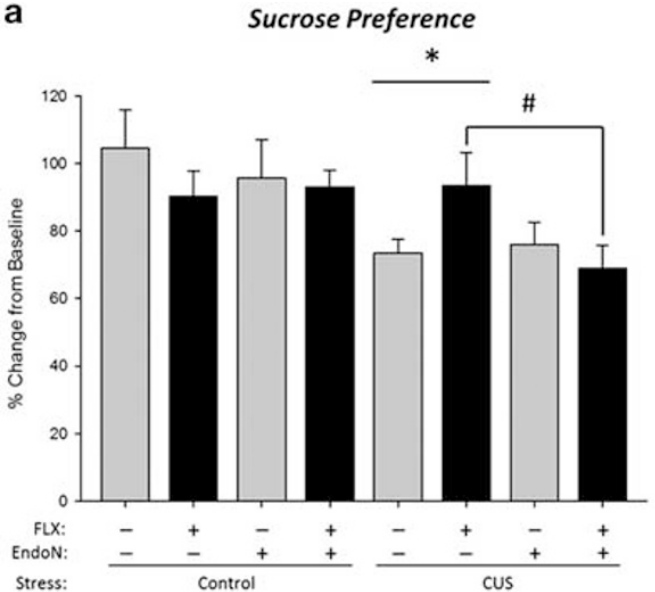

b

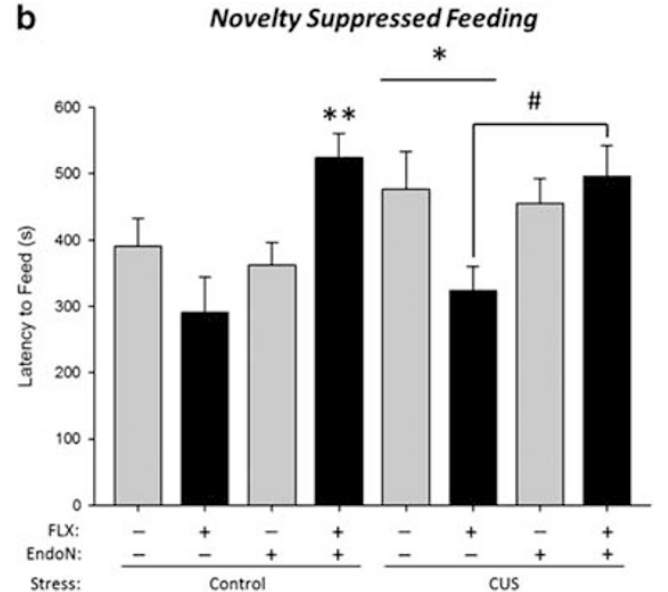

C

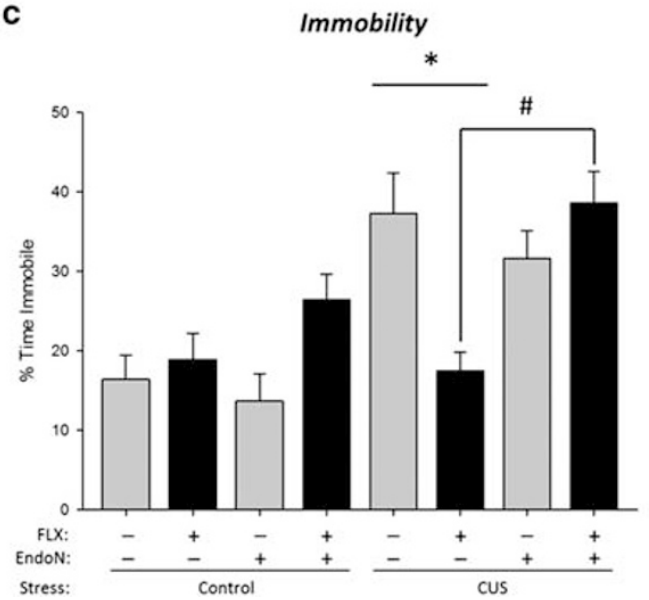

Forced Swim Test

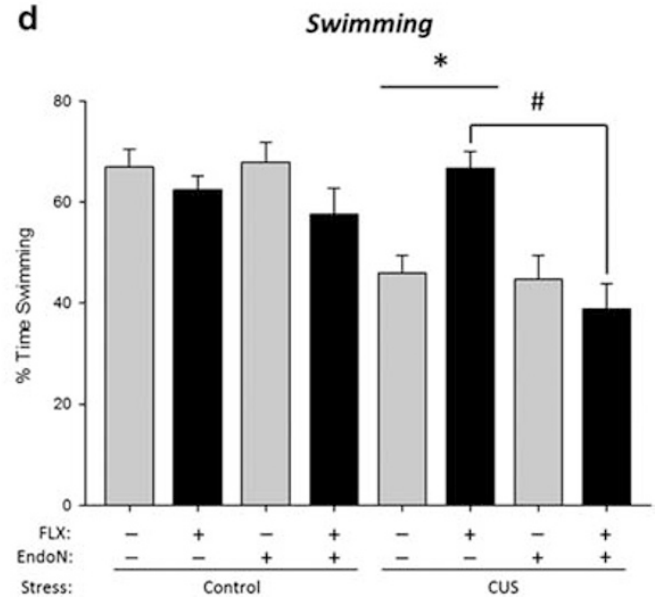

Figure 2 Endoneuraminidase $N$ (EndoN) inhibits antidepressant efficacy of fluoxetine (FLX) following chronic unpredictable stress (CUS)-induced behavioral changes. (a) Anhedonia was assessed in the sucrose preference test. Mean + SEM sucrose preference was reduced with CUS, FLX increased sucrose preference but this effect was inhibited by EndoN treatment $(* p<0.05$, \#p<0.04). (b) Anxiety- and depressive-like behavior was assessed in the novelty-suppressed feeding test as Mean + SEM latency to feed in seconds. In the CUS-exposed rats FLX decreased latency to feed, whereas EndoN treatment inhibited the effects of FLX to reduce latency to feed ( $* p<0.03$. $* * *<0.008, \# p<0.0005$ ). (c and d) Learned helplessness was evaluated in the forced swim test. Mean + SEM immobility was increased after CUS exposure, FLX decreased percentage time spent immobile, whereas EndoN inhibited FLX effects $(* p<0.04, \# p<0.00$ I). (d) Mean + SEM swimming behavior was decreased after CUS, whereas FLX increased but treatment with Endo inhibited the effects of FLX on percent time spent swimming (* $p<0.04$. \#p<0.0005). $n=7-9$ per group.

EndoN reduced PSD-95 expression in all three regions (Figure 4a-e; main effect of enzyme $p=0.018$ ). FLX increased PSD-95 expression under CUS condition only, regardless of region (stress by drug interaction $(p=0.009)$. A priori, EndoN treatment was expected to disrupt the effects of FLX, and indeed EndoN treatment attenuated the enhancement of PSD-95 expression by FLX in both the CA3 $(p=0.005)$ and DG $(p=0.011)$ regions, but not in the CA1 region $(p=0.44)$ under CUS conditions. There was also a main effect of region $(p<0.0001)$ but no other significant main effects or interactions ( $p$ 's $>0.06$ ).

\section{EndoN Treatment Attenuated CUS-Induced Increase in Basal Corticosterone Levels in the Saline-Treated Animals. FLX Decreased Testosterone Levels}

CUS increased basal CORT levels (weeks 3 and 6) regardless of drug or enzyme treatment (Figure 5a; stress by week interaction: $p<0.0002)$. EndoN significantly reduced basal
CORT at week $3(p<0.012)$, regardless of stress treatment, under vehicle but not FLX treatment (weeks by enzyme by drug interaction: $p<0.03$ ); however, it is important to note that FLX treatment had not been initiated yet. There was also a trend for a four-way interaction (weeks by enzyme by drug by stress with $p<0.064)$ and main effects of stress $(p<0.0001)$ and drug $(p<0.047)$, but no other significant effects $(p$ 's $>0.10)$. FLX treatment decreased testosterone levels, regardless of group (Figure $5 \mathrm{~b} p<0.034$; main effect of drug: $\mathrm{F}(1,47)=4.73, p=0.034)$, but no other significant main or interaction effects ( $p$ 's $>0.27$ ).

\section{DISCUSSION}

We found that PSA-NCAM is required for the behavioral antidepressant efficacy of FLX and necessary for multiple facets of FLX-induced neuroplasticity, but not the effects of FLX on endocrine measures. EndoN, which selectively 
cleaved PSA from NCAM, inhibited the antidepressant effects of FLX in each behavioral measure and attenuated the effects of FLX on synaptic protein expression, activation of neurons, and neurogenesis. Thus, the proper expression of PSA-NCAM is essential to the generation of antidepressant effects of FLX.

a

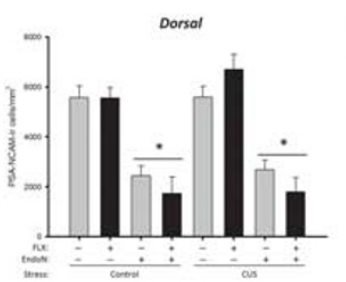

\section{CUS Induced a Depressive-Like Phenotype that was Reversed by FLX}

Our CUS protocol was successful in developing behavioral, endocrine, somatic, and neural depression-like endophenotypes consistent with the literature (Vollmayr et al, 2007; Willner, 2005). Rats exposed to CUS showed: (1) decreased

b

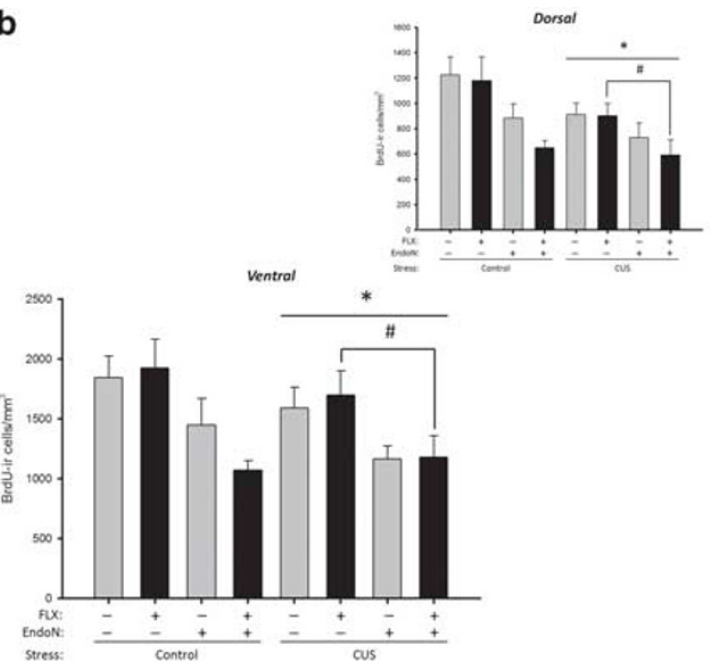

d
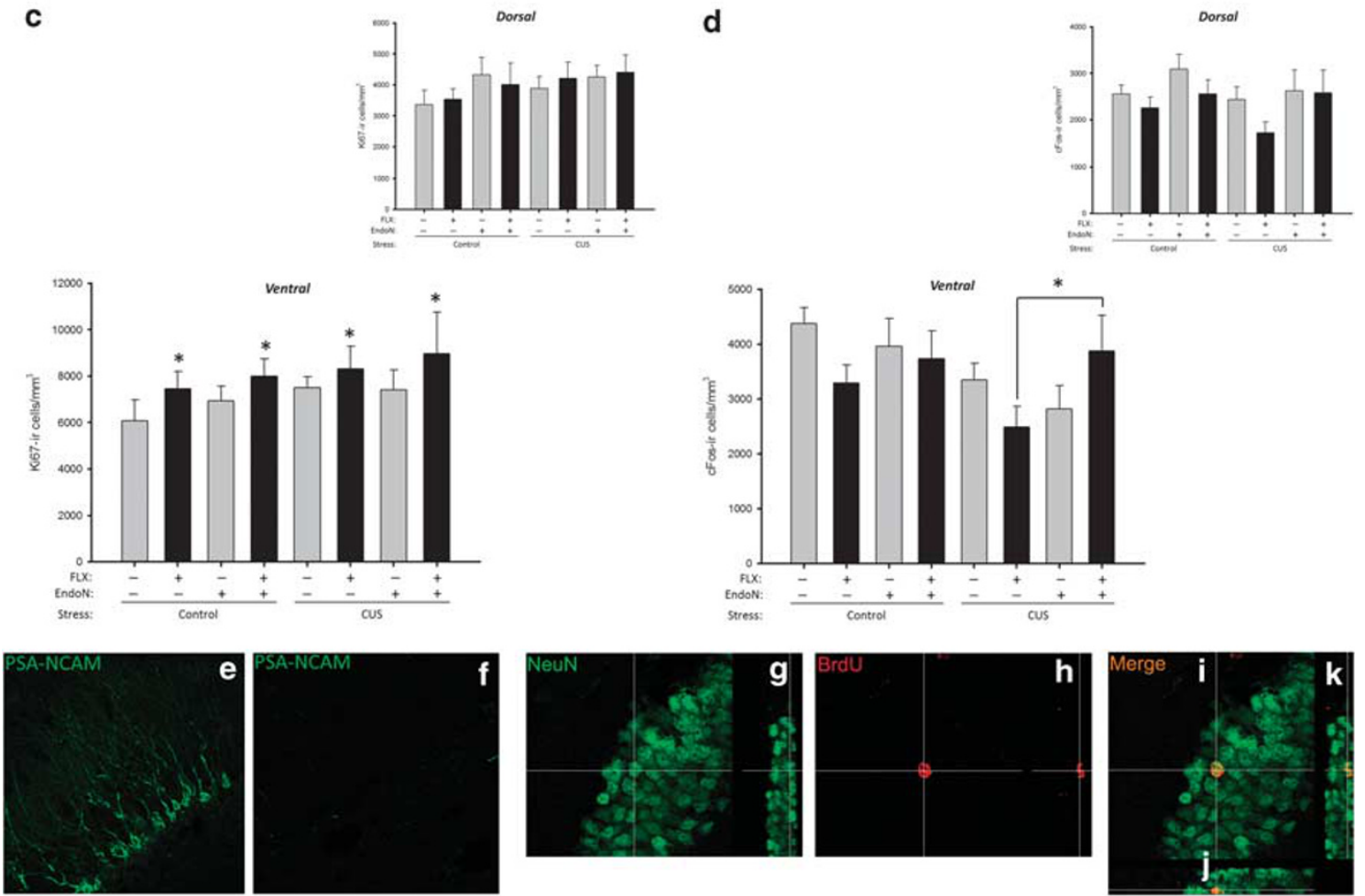

Figure 3 Endoneuraminidase N (EndoN) attenuates some measures of neuroplasticity following fluoxetine (FLX) treatment and chronic unpredictable stress (CUS). (a) Mean + SEM density of PSA-NCAM-ir cells in the granule cell layer (GCL). EndoN reduced PSA-NCAM-ir cells in the dentate gyrus regardless of CUS or FLX (*p $<0.0005)$. (b) Mean \pm SEM density of BrdU-ir cells in the GCL. EndoN and CUS reduced neurogenesis in the ventral GCL. However, FLX treatment reduced neurogenesis when given to the EndoN treated group (*p<0.04, \#p<0.04). (c) Mean + SEM density of Ki67-ir cells in the GCL. FLX treatment increased cell proliferation (Ki67-ir cells) in the ventral region compared with VEH treatment, regardless of group (*⿻ $p<0.04$ ). (d) Mean + SEM density of cFos-ir cells in the GCL. Expression of cFos-ir cells was reduced in the CUS groups after acute swim stress $(p<0.05)$, whereas under CUS EndoN reversed the FLX-induced decrease in cFos-ir cells $(* p<0.03)$. (e-i) Photomicrographs of (e) PSA-NCAM in vehicle treated and $(f)$ EndoN treated groups with a fluorescent secondary antibody and taken with a confocal microscope for improved clarity and contrast. Photomicrographs of (g) NeuN-ir cells in the GCL, (h) BrdU-ir cells in the GCL and (i) merged BrdU/NeuN-ir cell with confocal stacks in the $x-z$ plane (j) and in the $y-z$ plane (k). $n=6-10$ per group. 
PSD-95

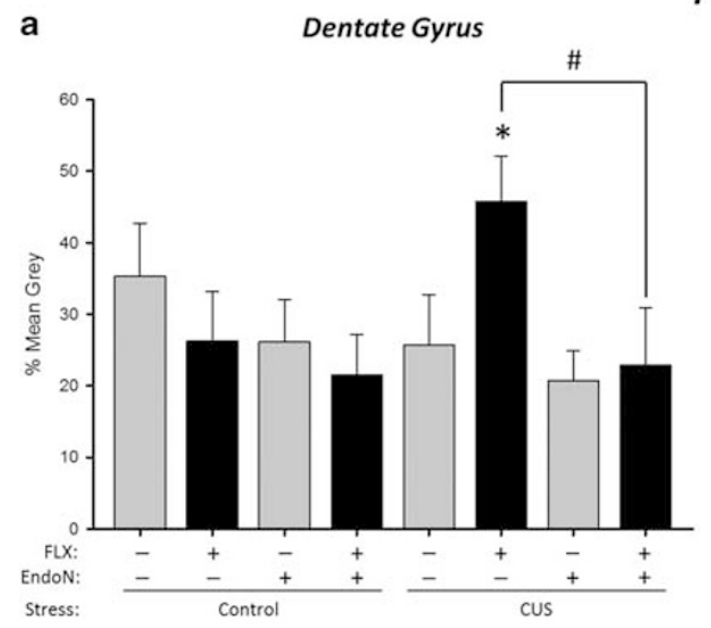

C

CA1

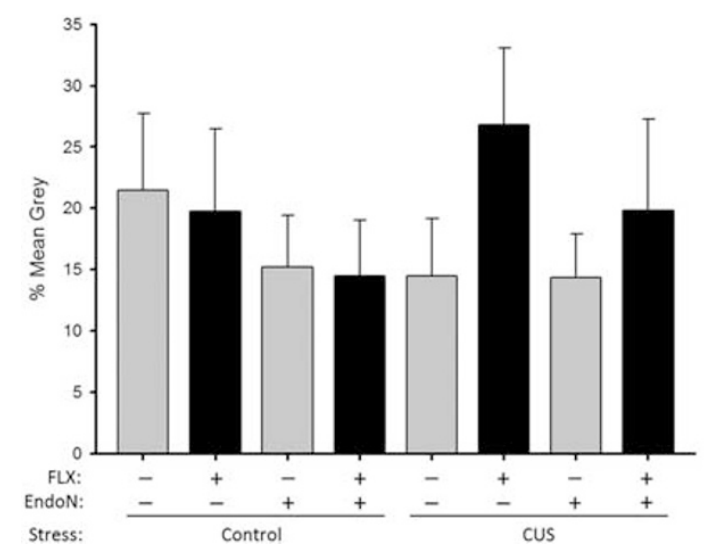

b
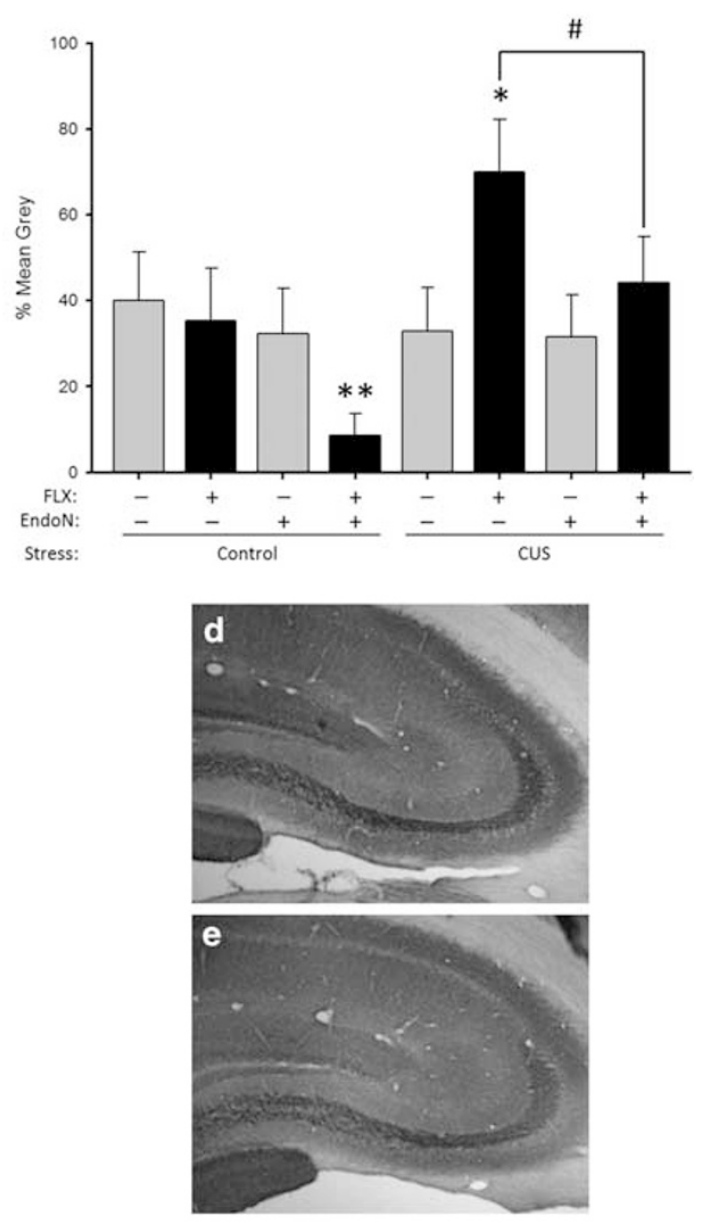

Figure 4 Endoneuraminidase $N($ EndoN) attenuates the fluoxetine ( $F L X)$ stimulation of PSD-95 expression in the dentate gyrus and CA3, but not CAI. Mean + SEM grey values for optical density of PSD-95 expression. (a) Optical density was increased by FLX in CUS-exposed rats, an effect inhibited by EndoN $(* p<0.009$, \#p<0.03). (b) Optical density was increased by FLX in CUS-exposed rats, an effect inhibited by EndoN treatment in CA3. EndoN treatment also decreased optical density in control rats $(* p<0.009$, $* * p<0.05, \# p<0.03$ ). (c) No significantly treatment effects on optical density were observed in CAI. $n=7-9$ per group. Photomicrograph of PSD-95 expression in the CA3 region of a FLX-injected rat exposed to CUS (d) and EndoN-infused rat given FLX and exposed to CUS (e). CUS, chronic unpredictable stress.

sucrose preference representative of anhedonia, (2) increased immobility in the forced swim test, indicative of increased passive stress-coping behavior, (3) increased basal CORT levels, (4) decreased hippocampal neurogenesis (BrdU/NeuN expression), and (5) decreased synaptic density (PSD-95 expression). CUS decreased cFos expression in the dentate gyrus in response to acute stress. Our results also demonstrate a replication of the well-established antidepressant effects of chronic FLX treatment within both behavioral and neural measures. In brief, FLX reversed CUS-induced anhedonia in the sucrose preference test, learned helplessness in the forced swim test, anxiety-like behavior in the noveltysuppressed feeding test. In addition, FLX increased hippocampal neurogenesis, PSD-95 expression, and PSA-NCAM expression. Having established a viable animal model of depression encompassing numerous endophenotypes and replicating the well-defined antidepressant effects of FLX, we were able to successfully determine the role of PSA-NCAM in antidepressant efficacy utilizing EndoN.

\section{EndoN Eliminated the Behavioral Efficacy of FLX}

The infusion of EndoN prevented the FLX-mediated increase in sucrose preference in CUS-exposed rats, thereby functionally disrupting antidepressant action. The antidepressant effects of FLX on sucrose preference are mediated by the $\mathrm{D}_{2}$-receptor (Muscat et al, 1992) and are unaltered by disruptions to hippocampal neurogenesis (Bessa et al, 2009; David et al, 2009). Therefore, the disruption of antidepressant efficacy by EndoN treatment is likely via the disruption of $\mathrm{D}_{2}$ receptor expression resultant of attenuated synaptic plasticity. There is a known PSA-NCAM- $\mathrm{D}_{2}$ receptor interaction, as $\mathrm{D}_{2}$ receptor activation increases PSA-NCAM expression in the medial prefrontal cortex (mPFC) (CastilloGomez et al, 2008), whereas EndoN treatment attenuates $\mathrm{D}_{2}$-mediated plasticity in the MPFC (Castillo-Gomez et al, 2011). Together these studies, along with the present findings, suggest an essential role for PSA-NCAM in the dopamine-mediated antidepressant effects of FLX, which serve to reduce anhedonia. 

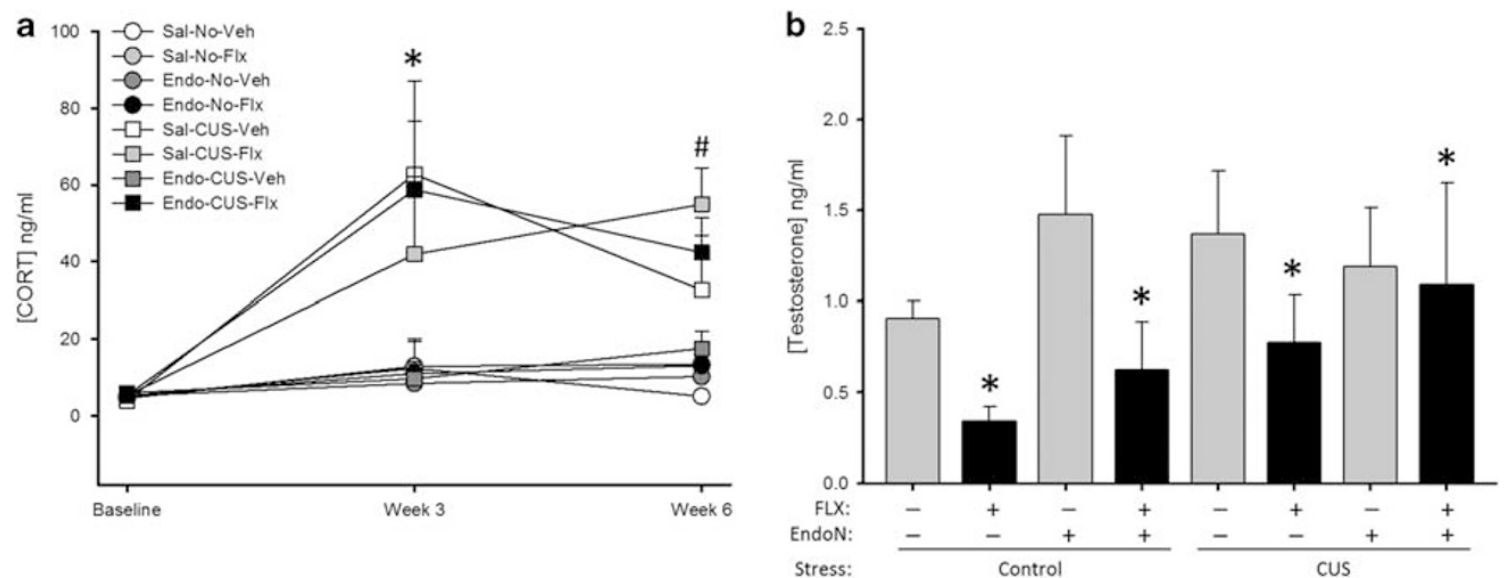

Figure 5 Chronic unpredictable stress (CUS) increased basal corticosterone level, and fluoxetine (FLX) reduced serum testosterone levels. (a) Mean + SEM basal serum corticosterone level. As expected, CUS exposure increased basal corticosterone; however, FLX failed to reduce basal corticosterone levels and EndoN did not significantly attenuate FLX effects (*p $<0.0002$, $\# p<0.03$ ). (b) Mean + SEM serum testosterone level. Regardless of group, FLX reduced testosterone levels. $(* p<0.04) . n=6-8$ per group.

In the forced swim test, infusion of EndoN prevented the FLX-mediated decrease in immobility, and increase in swimming, in CUS-exposed rats. Serotonergic antidepressants increase swimming behavior in the forced swim test, which is dependent on activation of the $5-\mathrm{HT}_{1 \mathrm{~A}}$ receptor (Detke et al, 1995; Redrobe et al, 1996), and swimming behavior is not affected by reductions in neurogenesis (Bessa et al, 2009; David et al, 2009). Thus, our findings suggest that the depletion of PSA-NCAM, via EndoN, interferes with appropriate serotonergic neurotransmission subsequently altering stress-coping behaviors in the forced swim test. Indeed, PSA-NCAM expression in the hippocampus is directly modulated by the $5-\mathrm{HT}_{1 \mathrm{~A}}$ receptor, and conversely PSA-NCAM may have a role in regulating 5 -HT receptor expression in the raphe nucleus (Black et al, 2009; Brezun and Daszuta, 2000; Grzegorzewska et al, 2010). The prevention of increased swimming by EndoN treatment is therefore likely indicative of altered serotonergic neurotransmission via the $5-\mathrm{HT}_{1 \mathrm{~A}}$ receptor. The glucocorticoid receptor is also important in the FLX-induced reduction in immobility (Lee et al, 2014) and glucocorticoid receptor activation is linked to hippocampal PSA-NCAM expression (Rodriguez et al, 1998). Our findings therefore demonstrate an essential role for PSA-NCAM in the antidepressant effects of FLX, potentially achieved through the modulation of serotonergic neurotransmission and modulation of glucocorticoid receptor activity, likely via downstream effects on neuroplasticity.

EndoN also prevented the FLX-mediated reduction in latency to feed in the novelty-suppressed feeding test, representing a disruption of neurogenesis-dependent antidepressant effects as attenuation of hippocampal neurogenesis also eliminates the ability of FLX to reduce the latency to feed (Bessa et al, 2009; David et al, 2009; Santarelli et al, 2003). It is possible that the effect of PSA-NCAM cleavage on novelty-induced hypophagia is due to a direct disruption of hippocampal neurogenesis, as found in the present study. Alternately, the depletion of PSA-NCAM may produce downstream effects via disrupted serotonergic neurotransmission, which regulates adult hippocampal neurogenesis through the $5-\mathrm{HT}_{1 \mathrm{~A}}$ receptor (Banasr et al, 2004; Radley and
Jacobs, 2002). Interestingly, EndoN treatment also disrupted the antidepressant effect of FLX in the noCUS treatment group, causing a significant increase in latency to feed. Acute treatment with FLX is anxiogenic (Silva et al, 1999), suggesting the ablation of PSA-NCAM and attenuation of the antidepressant effects of FLX may facilitate underlying anxiogenic properties. Taken together, our behavioral findings show that the ablation of PSA-NCAM, via EndoN treatment, impedes antidepressant efficacy in behavioral measures dependent on neurogenesis (novelty-suppressed feeding test) and behavioral measures independent of alterations in neurogenesis (sucrose preference and forced swim test), which instead, rely upon the modulation of monoaminergic neurotransmission.

\section{EndoN Reduced Some, but not all, Markers of Neuroplasticity in the Hippocampus}

The infusion of EndoN functioned as intended, greatly reducing the expression of PSA-NCAM 7-weeks after administration. This is partially consistent with previous reports showing that a much lower dose of EndoN than used in the present study (2000 times dilution) reduced PSA-NCAM expression for 4 weeks but was restored by 6 weeks in juvenile rats (Seki and Rutishauser, 1998). Our findings also show attenuated neuroplasticity, including the abatement of adult hippocampal neurogenesis by EndoN treatment, seen as a reduction in the number of BrdU-ir cells and BrdU/NeuN co-labeled cells 7-weeks after EndoN treatment, though cell proliferation (Ki67) was unaltered. This finding somewhat contrasts a previous report showing an increase in the proportion of BrdU/NeuN co-labeled cells, though cell proliferation and the number of 1-week old BrdU-ir cells were not significantly affected by EndoN treatment (Burgess et al, 2008). However, the length of the present study (53 days) greatly exceeds the previous report of 7-days, and suggests that EndoN reduces long-, but not short-term, survival of new neurons. EndoN reduced the expression of PSD-95 in the dentate gyrus and CA3 regions and to a lesser extent CA1, a finding indicative of reduced 
NMDA receptor expression and excitatory synaptic density (El-Husseini et al, 2000; Lin et al, 2006).

\section{EndoN Disrupted Some, but not all, Effects of FLX on Neuroplasticity Following CUS}

EndoN infusion attenuated the effects of FLX on hippocampal neuroplasticity, including measures of synaptic density (PSD-95 expression), neural activation (cFos expression), and neurogenesis. FLX treatment increased PSD-95 expression in the stratum lucidum of CA3 and the dentate gyrus in a manner consistent with the literature, which shows increased synaptic density following antidepressant treatment in both regions (Reines et al, 2008). EndoN attenuated this FLX-induced enhancement of PSD-95 expression in CA3 and dentate gyrus. This is the first finding demonstrating the inhibition of the antidepressantinduced enhancement of synaptic density via the selective depletion of PSA-NCAM. Disruption of the behavioral antidepressant efficacy of FLX coinciding with the inhibition of the FLX-induced upregulation of structural remodeling observed in our study, is consistent with previous findings showing an essential role of such plasticity in antidepressant efficacy (Bessa et al, 2009).

Following acute stress, cFos expression in the dentate gyrus was increased in the noCUS groups compared with the CUS groups. Lower cFos expression in rats exposed to CUS implies a reduced level of neural activity likely due to habituation of the stress-response in the CUS rats (Willner, 1997). Interestingly, FLX treatment decreased cFos expression regardless of stress, which may be representative of facilitated HPA-negative feedback with FLX treatment (Lee et al, 2014). Importantly, EndoN inhibited the effects of FLX, but only in the CUS-exposed group, serving to effectively increase neural activation in response to acute stress to the level of the noCUS groups.

Finally, it should be noted that EndoN was administered ICV, and though we restricted our measures of neuroplasticity to the hippocampus, EndoN would undoubtedly have affected neuroplasticity elsewhere in the brain. Future studies could examine other regions affected by depression or altered by chronic antidepressant treatment, such as the prefrontal cortex, dorsal raphe nucleus, and/or the amygdala. Such studies would determine whether the attenuation of PSA-NCAM mediated neuroplasticity via EndoN treatment in structures other than the hippocampus alters the development of CUS-induced depressive-like endophenotypes or antidepressant efficacy.

\section{EndoN Treatment did not Eliminate FLX Effects on Endocrine Measures}

Unlike both behavioral and neural responses to CUS, EndoN did not significantly influence basal CORT or testosterone levels in response to FLX or CUS. We did find an effect of EndoN to reduce basal CORT under vehicle, but not FLX conditions, on week 3 of CUS. However, given that FLX was not administered until week 3 this is likely a spurious finding and thus we contend that EndoN does not influence basal CORT. In the present study we found FLX treatment reduced serum testosterone levels but there was no significant effect of FLX to reduce basal CORT after CUS consistent with previous reports, (Bataineh and Daradka, 2007). These findings suggest that while EndoN serves to mediate the behavioral efficacy of FLX, it failed to affect changes to the gonadal axis in response to FLX or CUS.

\section{CONCLUSIONS}

Our findings demonstrate that the depletion of PSA-NCAM inhibits the behavioral efficacy of FLX and some aspects of FLX-induced neuroplasticity, showing PSA is required for antidepressant action. Given the role of PSA-NCAM in modulating a broad range of plasticity, this glycoprotein functions at the crux of multiple antidepressant mechanisms, and subsequently antidepressant efficacy. Thus, PSA-NCAM represents an interesting target for future research into the development of novel pharmacotherapies.

\section{FUNDING AND DISCLOSURE}

This work was funded by a Coast Capital Depression Fund grant and Canadian Institutes of Health Research grant (MOP142308) to LAMG. JRE and CKB were funded by Michael Smith Graduate Awards. The authors declare no conflicts of interest.

\section{ACKNOWLEDGMENTS}

We thank the animal care staff at the Department of Psychology for their assistance.

\section{REFERENCES}

Anisman H, Zacharko RM (1982). Depression: the predisposing influence of stress. Behav Brain Sci 5: 89-99.

Aonurm-Helm A, Jurgenson M, Zharkovsky T, Sonn K, Berezin V, Bock E et al (2008). Depression-like behaviour in neural cell adhesion molecule (NCAM)-deficient mice and its reversal by an NCAM-derived peptide, FGL. Eur J Neurosci 28: 1618-1628.

Banasr M, Hery M, Printemps R, Daszuta A (2004). Serotonininduced increases in adult cell proliferation and neurogenesis are mediated through different and common 5-HT receptor subtypes in the dentate gyrus and the subventricular zone. Neuropsychopharmacology 29: 450-460.

Bataineh HN, Daradka T (2007). Effects of long-term use of fluoxetine on fertility parameters in adult male rats. Neuro Endocrinol Lett 28: 321-325.

Bessa JM, Ferreira D, Melo I, Marques F, Cerqueira JJ, Palha JA et al (2009). The mood-improving actions of antidepressants do not depend on neurogenesis but are associated with neuronal remodeling. Mol Psychiatry 14: 764-773 739.

Black MA, Deurveilher S, Seki T, Marsh DR, Rutishauser U, Rafuse VF et al (2009). Role of polysialylated neural cell adhesion molecule in rapid eye movement sleep regulation in rats. Eur $J$ Neuroscience 30: 2190-2204.

Boldrini M, Underwood MD, Hen R, Rosoklija GB, Dwork AJ, John Mann J et al (2009). Antidepressants increase neural progenitor cells in the human hippocampus. Neuropsychopharmacology 34: 2376-2389.

Brezun JM, Daszuta A (2000). Serotonergic reinnervation reverses lesion-induced decreases in PSA-NCAM labeling and proliferation of hippocampal cells in adult rats. Hippocampus 10: 37-46.

Burgess A, Wainwright SR, Shihabuddin LS, Rutishauser U, Seki T, Aubert I (2008). Polysialic acid regulates the clustering, migration, 
and neuronal differentiation of progenitor cells in the adult hippocampus. Dev Neurobiol 68: 1580-1590.

Byrne SE, Rothschild AJ (1998). Loss of antidepressant efficacy during maintenance therapy: possible mechanisms and treatments. J Clin Psychiatry 59: 279-288.

Castillo-Gomez E, Gomez-Climent MA, Varea E, Guirado R, Blasco-Ibanez JM, Crespo C et al (2008). Dopamine acting through D2 receptors modulates the expression of PSA-NCAM, a molecule related to neuronal structural plasticity, in the medial prefrontal cortex of adult rats. Exp Neurol 214: 97-111.

Castillo-Gomez E, Varea E, Blasco-Ibanez JM, Crespo C, Nacher J (2011). Polysialic acid is required for dopamine D2 receptormediated plasticity involving inhibitory circuits of the rat medial prefrontal cortex. PloS One 6: e29516.

Dantzer R, O'Connor JC, Freund GG, Johnson RW, Kelley KW (2008). From inflammation to sickness and depression: when the immune system subjugates the brain. Nat Rev Neurosci 9: 46-56.

David DJ, Samuels BA, Rainer Q, Wang JW, Marsteller D, Mendez I et al (2009). Neurogenesis-dependent and -independent effects of fluoxetine in an animal model of anxiety/depression. Neuron 62: 479-493.

Detke MJ, Rickels M, Lucki I (1995). Active behaviors in the rat forced swimming test differentially produced by serotonergic and noradrenergic antidepressants. Psychopharmacology 121: 66-72.

Egeland M, Warner-Schmidt J, Greengard P, Svenningsson P (2010). Neurogenic effects of fluoxetine are attenuated in p11 (S100A10) knockout mice. Biol Psychiatry 67: 1048-1056.

El-Husseini AE, Schnell E, Chetkovich DM, Nicoll RA, Bredt DS (2000). PSD-95 involvement in maturation of excitatory synapses. Science 290: 1364-1368.

Elsayed M, Banasr M, Duric V, Fournier NM, Licznerski P, Duman RS (2012). Antidepressant effects of fibroblast growth factor-2 in behavioral and cellular models of depression. Biol Psychiatry 72: 258-265.

Epp JR, Beasley CL, Galea LA (2013). Increased hippocampal neurogenesis and p21 expression in depression: dependent on antidepressants, sex, age, and antipsychotic exposure. Neuropsychopharmacology 38: 2297-2306.

Epp JR, Haack AK, Galea LA (2010). Task difficulty in the Morris water task influences the survival of new neurons in the dentate gyrus. Hippocampus 20: 866-876.

Fanselow MS, Dong HW (2010). Are the dorsal and ventral hippocampus functionally distinct structures? Neuron 65: 7-19.

Ferrari AJ, Charlson FJ, Norman RE, Patten SB, Freedman G, Murray CJ et al (2013). Burden of depressive disorders by country, sex, age, and year: findings from the global burden of disease study 2010. PLoS Med 10: e1001547.

Grzegorzewska M, Mackowiak M, Wedzony K, Hess G (2010). 5-HT1A receptors mediate detrimental effects of cocaine on longterm potentiation and expression of polysialylated neural cell adhesion molecule protein in rat dentate gyrus. Neuroscience 166: 122-131.

Hallenbeck PC, Vimr ER, Yu F, Bassler B, Troy FA (1987). Purification and properties of a bacteriophage-induced endo-Nacetylneuraminidase specific for poly-alpha-2,8-sialosyl carbohydrate units. J Biol Chem 262: 3553-3561.

Holsboer F (2000). The corticosteroid receptor hypothesis of depression. Neuropsychopharmacology 23: 477-501.

Ising M, Horstmann S, Kloiber S, Lucae S, Binder EB, Kern N et al (2007). Combined dexamethasone/corticotropin releasing hormone test predicts treatment response in major depression - a potential biomarker? Biol Psychiatry 62: 47-54.

Koo JW, Russo SJ, Ferguson D, Nestler EJ, Duman RS (2010). Nuclear factor-kappaB is a critical mediator of stress-impaired neurogenesis and depressive behavior. Proc Natl Acad Sci USA 107: 2669-2674.

Krushel LA, Cunningham BA, Edelman GM, Crossin KL (1999). NF-kappaB activity is induced by neural cell adhesion molecule binding to neurons and astrocytes. J Biol Chem 274: 2432-2439.

Lee MS, Kim YH, Park WS, Park OK, Kwon SH, Hong KS et al (2014). Temporal variability of glucocorticoid receptor activity is functionally important for the therapeutic action of fluoxetine in the hippocampus. Mol Psychiatry (doi:10.1038/mp.2014.137).

Lieben CK, Steinbusch HW, Blokland A (2006). 5,7-DHT lesion of the dorsal raphe nuclei impairs object recognition but not affective behavior and corticosterone response to stressor in the rat. Behav Brain Res 168: 197-207.

Lin Y, Jover-Mengual T, Wong J, Bennett MV, Zukin RS (2006). PSD-95 and PKC converge in regulating NMDA receptor trafficking and gating. Proc Natl Acad Sci USA 103: 19902-19907.

McCall T, Weil ZM, Nacher J, Bloss EB, El Maarouf A, Rutishauser U et al (2013). Depletion of polysialic acid from neural cell adhesion molecule (PSA-NCAM) increases CA3 dendritic arborization and increases vulnerability to excitotoxicity. Exp Neurol 241: 5-12.

Muller D, Wang C, Skibo G, Toni N, Cremer H, Calaora V et al (1996). PSA-NCAM is required for activity-induced synaptic plasticity. Neuron 17: 413-422.

Muscat R, Papp M, Willner P (1992). Reversal of stress-induced anhedonia by the atypical antidepressants, fluoxetine and maprotiline. Psychopharmacology 109: 433-438.

Radley JJ, Jacobs BL (2002). 5-HT1A receptor antagonist administration decreases cell proliferation in the dentate gyrus. Brain Res 955: 264-267.

Redrobe JP, MacSweeney CP, Bourin M (1996). The role of 5-HT1A and 5-HT1B receptors in antidepressant drug actions in the mouse forced swimming test. Eur J Pharmacology 318: 213-220.

Reines A, Cereseto M, Ferrero A, Sifonios L, Podesta MF, Wikinski S (2008). Maintenance treatment with fluoxetine is necessary to sustain normal levels of synaptic markers in an experimental model of depression: correlation with behavioral response. Neuropsychopharmacology 33: 1896-1908.

Rodriguez JJ, Montaron MF, Petry KG, Aurousseau C, Marinelli M, Premier $S$ et al (1998). Complex regulation of the expression of the polysialylated form of the neuronal cell adhesion molecule by glucocorticoids in the rat hippocampus. Eur J Neurosci 10: 2994-3006.

Rutishauser U (2008). Polysialic acid in the plasticity of the developing and adult vertebrate nervous system. Nat Rev Neurosci 9: 26-35.

Rutishauser U, Watanabe M, Silver J, Troy FA, Vimr ER (1985). Specific alteration of NCAM-mediated cell adhesion by an endoneuraminidase. J Cell Biol 101(5 Pt 1): 1842-1849.

Sairanen M, O'Leary OF, Knuuttila JE, Castren E (2007). Chronic antidepressant treatment selectively increases expression of plasticity-related proteins in the hippocampus and medial prefrontal cortex of the rat. Neuroscience 144: 368-374.

Santarelli L, Saxe M, Gross C, Surget A, Battaglia F, Dulawa S et al (2003). Requirement of hippocampal neurogenesis for the behavioral effects of antidepressants. Science 301: 805-809.

Sapolsky RM (1986). Glucocorticoid toxicity in the hippocampus: reversal by supplementation with brain fuels. J Neurosci 6: 2240-2244.

Seki T, Rutishauser U (1998). Removal of polysialic acid-neural cell adhesion molecule induces aberrant mossy fiber innervation and ectopic synaptogenesis in the hippocampus. J Neuroscience 18: 3757-3766.

Silva MT, Alves CR, Santarem EM (1999). Anxiogenic-like effect of acute and chronic fluoxetine on rats tested on the elevated plusmaze. Braz J Med Biol Res 32: 333-339.

Snyder JS, Clifford MA, Jeurling SI, Cameron HA (2012). Complementary activation of hippocampal-cortical subregions and immature neurons following chronic training in single and multiple context versions of the water maze. Behav Brain Res 227: 330-339. 
Stockmeier CA, Mahajan GJ, Konick LC, Overholser JC, Jurjus GJ, Meltzer HY et al (2004). Cellular changes in the postmortem hippocampus in major depression. Biol Psychiatry 56: 640-650.

Varea E, Blasco-Ibanez JM, Gomez-Climent MA, Castillo-Gomez E, Crespo C, Martinez-Guijarro FJ et al (2007). Chronic fluoxetine treatment increases the expression of PSA-NCAM in the medial prefrontal cortex. Neuropsychopharmacology 32: 803-812.

Vollmayr B, Mahlstedt MM, Henn FA (2007). Neurogenesis and depression: what animal models tell us about the link. Eur Arch Psychiatry Clin Neurosci 257: 300-303.

Vutskits L, Djebbara-Hannas Z, Zhang H, Paccaud JP, Durbec P, Rougon $\mathrm{G}$ et al (2001). PSA-NCAM modulates BDNF-dependent survival and differentiation of cortical neurons. Eur J Neurosci 13: 1391-1402.
Wainwright SR, Galea LA (2013). The neural plasticity theory of depression: assessing the roles of adult neurogenesis and PSA-NCAM within the hippocampus. Neural Plast 2013: 805497.

Wainwright SR, Lieblich SE, Galea LA (2011). Hypogonadism predisposes males to the development of behavioural and neuroplastic depressive phenotypes. Psychoneuroendocrinology 36: $1327-1341$.

Willner P (1997). Validity, reliability and utility of the chronic mild stress model of depression: a 10-year review and evaluation. Psychopharmacology 134: 319-329.

Willner P (2005). Chronic mild stress (CMS) revisited: consistency and behavioral-neurobiological concordance in the effects of CMS. Neuropsychobiology 52: 90-110. 